\title{
Interfaces in the Potts Model II: Antonov's Rule and Rigidity of the Order Disorder Interface
}

\author{
Alain Messager, Salvador Miracle-Sole, Jean Ruiz and Senya Shlosman \\ Centre de Physique Théorique* ${ }^{\star \star}$, CNRS-Luminy-Case 907, F-13288 Marseille Cedex 9, France
}

Received August 1, 1990

\begin{abstract}
Within the ferromagnetic $q$-state Potts model we discuss the wetting of the interface between two ordered phases $a$ and $b$ by the disordered phase $f$ at the transition temperature. In two or more dimensions and for $q$ large we establish the validity of the Antonov's rule, $\sigma^{a b}=\sigma^{a f}+\sigma^{f b}$, where $\sigma$ denotes the surface tension between the considered phases. We also prove that at this temperature, in three or more dimensions the interface between any ordered phase and the disordered one is rigid.
\end{abstract}

\section{Introduction}

In recent years considerable progress has been achieved on the study of the physical properties of the interfaces between two coexisting phases. When three or more phases coexist new physical phenomena appear whose understanding requires the study of several interacting interfaces.

A simple model in which this situation occurs in the ferromagnetic $q$-state Potts model in dimension $d \geqq 2$. This model exhibits $q$ ordered phases at low temperatures and one disordered phase at high temperatures. For $q$ large enough, the order-disorder transition is first order, and all the previous phases coexist and are distinct at some unique transition inverse temperature $\beta_{t}$. For rigorous results, see $[1-6]$.

In the first part of this work [6] we have presented an analysis of the order-disorder transition for large $q$ based on an adaptation of the theory of Pirogov and Sinaï to the Fortuin-Kasteleyn representation of the Potts model. In the present paper we develop this approach, and apply it to study the behaviour

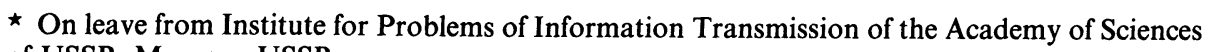
of USSR, Moscow, USSR

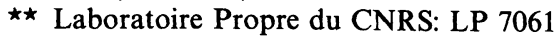


of the different interfaces which occur at the transition point. Different aspects of this problem have also been discussed in [7-11].

For this purpose we shall, as usually, enforce two phases, say $\alpha$ and $\beta$, to coexist, by considering an asymmetric boundary condition, i.e. the condition corresponding to $\alpha$ on the top half and the condition corresponding to $\beta$ on the lower half of a large rectangular box.

The surface tension $\sigma^{\alpha \beta}$, that is the residual free energy associated to the presence of an interface between the phases $\alpha$ and $\beta$, is then defined by taking the difference between the free energy of the above described system and the average of the free energies corresponding to the homogeneous boundary conditions $\alpha$ and $\beta$.

Due to the symmetry of the model, all ordered phases may be regarded as equivalent and we are lead to consider two kinds of phase coexistence: the coexistence between an ordered phase and the disordered phase and the coexistence between two ordered phases; previous results on these surface tensions were obtained in [4-5].

In the first case, as a result of our analysis, we obtain a description of the interface very close, for $q$ large enough, to that of the plus-minus interface for the Ising ferromagnet at low temperature [12]. Namely, the order disorder interface may be expressed as a gas of elementary excitations with small activities. These elementary excitations are the components of the deformations with respect to the interface of the ground state. As an application of this result we prove the rigidity of the order disorder interface in dimension $d \geqq 3$ for $q$ large enough.

In the second case, we show that the boundary between the two ordered phases is described by two interacting interfaces. In the region between these interfaces the system is submitted to the boundary conditions associated to the disordered phase. If we consider each of them separately, these interfaces admit a representation very similar to that described in the previous paragraph, but they strongly interfact if they are at short distances.

Using these representations of the interfaces and the mathematical definition of the surface tensions given above we are then able to prove, at the transition temperature $\beta_{t}$, the following relation

$$
\sigma^{a b}=\sigma^{a f}+\sigma^{f b}
$$

where $a, b$ and $f$ denote two different ordered and the disordered phases. Such a relation among surface tensions is known as Antonov's rule. We notice that an essential step in the proof, the inequality $\sigma^{a b} \geqq \sigma^{a f}+\sigma^{f b}$, was already derived in [10]. This inequality reflects the specific features of the Potts model. On the other hand, the inequality, $\sigma^{\alpha \gamma} \leqq \alpha^{\alpha \beta}+\sigma^{\beta \gamma}$, where $\alpha, \beta, \gamma$ are any three different coexisting phases of the system seems to be of a very general nature. We pass now to give the rough idea of why it is so.

To estimate the surface tension $\sigma^{\alpha \gamma}$ from above we have to estimate from below the partition function $Z_{L}^{\alpha \gamma}$ in a volume of size $L$ with mixed $\alpha-\gamma$ boundary conditions. We do this by restricting the summation to the configurations which have the strip of intermediate phase $\beta$, inserted between $\alpha$ and $\gamma$, of thickness $L^{p}$. If $p$ is less than 1 , then the boundary effects of interacting of $\beta$ phase with spins $\alpha$ or $\gamma$ along the boundary of our volume, does not contribute to the surface term. On the other hand, if the size of fluctuations of the $\alpha-\beta$ and $\beta-\gamma$ interfaces are of order $L^{q}$ with 
$q<p$, then these surfaces essentially do not interact, and so their surface free energies per unit area is $\sigma^{\alpha \beta}, \sigma^{\beta \gamma}$, so the total contribution to the free energy of the $\alpha-\gamma$ interface is at least $\sigma^{\alpha \beta}+\sigma^{\beta \gamma}$. Below we prove exactly that kind of statement for the 2-dimensional Potts model with $p=2 / 3, q=1 / 2+\varepsilon$, and for the 3-dimensional Potts model with $q$ arbitrary small and $p>q$.

Since in the Potts model at the transition temperature the ordered phases $a$, $b$ and the disordered phase $f$ are in equilibrium, Antonov's rule implies that there is no variation of the free energy to prevent a layer of the disordered phase, between two ordered phases, for becoming macroscopic. This is the (perfect or complete) wetting phenomenon. Thus we conclude that the thermodynamic condition for wetting is fulfilled in our case.

In order to complete the theory, an interesting effort would be to study the width of the intermediate layer using statistical mechanical arguments. One believes that this layer is macroscopic (in other words, that the width tends to infinity with the size of the box) as a result of an "entropic repulsion" between the two interfaces. Distant interfaces, having more freedom to fluctuate, should be more likely. Instead, for $\beta>\beta_{t}$, a macroscopic layer of disordered phase cannot exist and the intermediate region should have a finite thickness. Then the question arises of how does this thickness grow when $\beta$ approaches $\beta_{t}$. The thickness is expected to diverge, as predicted by mean field theory or numerical experiments, and for $d \geqq 3$ (rigid interfaces) this occurs through an infinite sequence of layering transitions. We notice that entropic repulsion and layering have indeed been proved in the case of an SOS surface on a wall [13]. For more details and references concerning these phenomena we refer the reader to [11], especially to Chap. VI.

\section{Convergent Expansions}

We consider the $d$-dimensional cubic lattice $\mathbb{Z}^{d}$ and denote by $\mathscr{B}$ the set of bonds of the lattice (pairs of nearest neighbours). In the Potts model spin variables $\sigma_{i}$ which take values on a discrete set $\{1,2, \ldots q\}$ are associated with each site $i$ of the lattice. The interaction energy is

$$
H=-\sum \delta\left(\sigma_{i}, \sigma_{j}\right)
$$

where the sum runs over pairs of nearest neighbours and $\delta$ is the Kronecker symbol (we take the interaction potential equal to 1 ).

We shall use in the present work an alternative representation of this model, due to Fortuin and Kasteleyn, where the configurations of the system are associated to subsets $X \subset \mathscr{B}$ of occupied bonds. The Boltzmann factors and the partition functions take then the form which will be given below, and $q$ may be real valued.

Two bonds are called adjacent if they have an endpoint in common. Two bonds are called co-adjacent if there exists a $d$-cell containing these two bonds ( $d$-cells are plaquettes for $d=2$, cubes for $d=3$, etc.). A set of bonds $X \subset \mathscr{B}$, is connected (respectively co-connected) if for any $b$ and $b^{\prime}$ in $X$, there is a sequence $b=b_{1}, b_{2}, \ldots, b_{n}=b^{\prime}$ of bonds in $X$ such that $b_{i}$ and $b_{i+1}$ are adjacent (respectively co-adjacent) for all $i=1, \ldots, n-1$.

For any set of bonds $X \subset \mathscr{B}$, we define the boundary $\partial X$ as the set of bonds which belong to $X$ and are co-connected to the complementary $X^{c}=\mathscr{B} / X$. We 
define the co-boundary $\delta X$ as the set of bonds which belong to $X^{c}$ and are connected to $X$. We denote by $S(X)$ the set of sites which belong to some bond in $X$. Then, $\delta X=\delta_{1} X \cup \delta_{2} X$, where $\delta_{n} X=\left\{b \in X^{c} /|S(b) \cap S(X)|=n\right\}, n=1,2$. Hereafter $|E|$ denotes the cardinality of the set $E$.

A co-connected subset $\gamma$ of $\mathscr{B}$ is called a contour if it is the co-boundary of some set $X \subset \mathscr{B}$, such that either $X$ or $X^{c}$ is a finite set. In the first case we say that $\gamma$ is a contour of the free class, or a $f$-contour, in the second case we say that $\gamma$ is a contour of the wired class, or a $w$-contour. The unique infinite component of $\mathscr{B} \backslash \gamma$ is denoted by Ext $\gamma$ and we define the sets $V(\gamma)=\mathscr{B} \backslash \operatorname{Ext} \gamma$, and Int $\gamma=V(\gamma) \backslash \gamma$ and the length of the contour

$$
\begin{array}{ll}
\|\gamma\|=\left|\delta_{1} \operatorname{Int} \gamma\right|+2\left|\delta_{2} \operatorname{Int} \gamma\right|, & \text { if } \gamma \text { is a } f \text {-contour, } \\
\|\gamma\|=\left|\delta_{1} \operatorname{Ext} \gamma\right|+2\left|\delta_{2} \operatorname{Ext} \gamma\right|, & \text { if } \gamma \text { is a } w \text {-contour. }
\end{array}
$$

Two contours $\gamma_{1}, \gamma_{2}$ are called mutually compatible if $\gamma_{1} \cup \gamma_{2}$ is not co-connected.

We introduce the partition functions with free and wired boundary conditions

$$
\begin{aligned}
& Z^{f}(V)=\sum_{X \subset V, X \cap \delta V^{c}=\varnothing}\left(e^{\beta}-1\right)^{|X|} q^{C_{V}(X)}, \\
& Z^{w}(V)=\sum_{X \subset V, X \supset \partial V}\left(e^{\beta}-1\right)^{|X|} q^{C_{V}(X)},
\end{aligned}
$$

where $V \subset \mathscr{B}$ and $C_{V}(X)$ denotes the number of connected components of $X$, counting each site of $S_{I}(V) \backslash S(X)$, where $S_{I}(V)=S(V) \backslash S\left(V^{c}\right)$, as a component. The following limits (independent of the boundary conditions):

$$
f(\beta)=\lim _{V \uparrow \mathscr{B}}(1 /|V|) \log Z^{f}(V)=\lim _{V \uparrow \mathscr{B}}(1 /|V|) \log Z^{w}(V),
$$

exist and give the free energy per bond: $-(1 / \beta) f(\beta)$.

We shall express the partition functions $Z^{w}$ and $Z^{f}$ in terms of two contour models associated with the functionals $\phi_{w}$ and $\phi_{f}$ defined respectively on the set of $w$-contours and the set of $f$-contours. The partition function, in a volume $V(V \subset \mathscr{B})$, of a contour model with contour weights $\phi(\gamma)$ is given by

$$
\mathscr{Z}(V \mid \phi)=\sum_{\partial \subset V} \prod_{\gamma \in \partial} \phi(\gamma)
$$

where the sum is over all admissible families $\partial$ of contours in $V$ : this will mean that $\partial_{w}$ is a set of mutually compatible $w$-contours included in $V \backslash \partial V$ and $\partial_{f}$ is a set of mutually compatible $f$-contours included in $V$.

To have a good control on contour models one relief and their " $\tau$-functionality." A contour functional $\phi$ is said to be a $\tau$-functional if it satisfies the estimate

$$
|\phi(\gamma)| \leqq \exp (-\tau\|\gamma\|)
$$

for some $\tau>0$ and every contour $\gamma$. This ensures, in particular, the existence of the limit

$$
f(\phi)=\lim _{V \uparrow \mathscr{B}}(1 /|V|) \log \mathscr{Z}(V \mid \phi)
$$

Proposition 1. Assume that $q$ is large enough and that $\beta$ is equal to $\beta_{t}=\beta_{t}(q, d)$ the inverse transition temperature. Then two $\tau$-functionals on the set of contour, $\phi_{w}$ 
and $\phi_{f}$ exist, such that:

$$
\begin{aligned}
& Z^{w}(V)=q^{C(V)}\left(e^{\beta}-1\right)^{|V|} \mathscr{Z}\left(V \mid \phi_{w}\right), \\
& Z^{f}(V)=q^{S_{I}(V)} \mathscr{Z}\left(V \mid \phi_{f}\right)
\end{aligned}
$$

with $\tau_{w}=\frac{1}{2 d} \log q-9 q^{-1 / 2 d}$ and $\tau_{f}=\frac{d-1}{d(4 d-2)} \log q-6 q^{-[(d-1) /(4 d-2) d]}$. Moreover

$$
f\left(\beta_{t}\right)=\log \left(e^{\beta_{t}}-1\right)+f\left(\phi_{w}\right)=(1 / d) \log q+f\left(\phi_{f}\right) .
$$

We refer the reader to [6] for the proof.

We now introduce clusters of contours in order to write the logarithm of the partition functions as a sum over these clusters. Let $C=\left\{\gamma_{1}, \gamma_{2}, \ldots, \gamma_{n}\right\}$ be a set of contours (of the same class) where $\gamma_{1}, \gamma_{2}, \ldots, \gamma_{n}$ are not necessarily mutually compatible. We say that the family $C$ is incompatible with the contour $\gamma$ and write $C i \gamma$, if there exists some $\gamma^{\prime}$ in $C$ such that $\gamma$ is not compatible with $\gamma^{\prime}$. The family $C$ is called a cluster if it can not be decomposed into two non-empty subsets $C_{1} \cup C_{2}$ such that all pairs $\gamma_{1}, \gamma_{2}$ with $\gamma_{1} \in C_{1}$ and $\gamma_{2} \in C_{2}$, are compatible contours. According to Kotecky and Preiss [14] we define on the set of clusters the truncated functional associated to a contour functional $\phi$ by

$$
\phi_{0}^{T}(C)=\sum_{B \subset C}(-1)^{N(C)-N(B)} \log \sum_{\partial \subset C} \prod_{\gamma \in \partial} \phi(\gamma),
$$

where $B$ and $C$ are arbitrary sets of contours, $N(C)$ and $N(B)$ the number of contours and $\partial$ are admissible families of compatible contours (then $\phi_{0}^{T}(C)=0$ whenever $C$ is not a cluster). Let $B(C)$ be the set of bonds $\gamma_{1} \cup \gamma_{2} \cup \cdots \cup \gamma_{n}$. The definitions above say that a set of contours $C$ is a cluster if $B(C)$ is a co-connected set and $C i \gamma$ means that $B(C) \cup \gamma$ is co-connected. It will be convenient in the following to consider a cluster just as a set of bonds and to change the definitions according to this point of view. We shall say therefore from now on, that $C$ is a cluster if $C \subset \mathscr{B}$ and it happens that $C=\gamma \cup \gamma_{2} \cup \cdots \cup \gamma_{n}$, where $C^{\prime}=\left\{\gamma_{1}, \gamma_{2}, \ldots, \gamma_{n}\right\}$ is a cluster in the sense of the old definition. We shall define $\|C\|=\left\|\gamma_{1}\right\|+\cdots+\left\|\gamma_{n}\right\|$ and the corresponding truncated functionals by

$$
\phi^{T}(C)=\sum_{C^{\prime}: B\left(C^{\prime}\right)=C} \phi_{0}^{T}\left(C^{\prime}\right)
$$

Using these definitions, we obtain as a consequence of Proposition 1 and Theorem 1 in [14]:

Proposition 2. Assume that $q$ is large enough and that $\beta$ is equal to $\beta_{t}$. Then two truncated functionals $\phi_{w}^{T}$ and $\phi_{f}^{T}$ exist, such that

$$
\begin{aligned}
q^{-1}\left(e^{\beta}-1\right)^{-|V|} Z^{w}(V) & =\exp \left\{\sum_{c_{w} \subset V} \phi_{w}^{T}\left(C_{w}\right)\right\}, \\
q^{-S_{I}(V)} Z^{f}(V) & =\exp \left\{\sum_{C_{f} \subset V} \phi_{f}^{T}\left(C_{f}\right)\right\} .
\end{aligned}
$$

They satisfy, for some $k>0$, the estimates

$$
\begin{aligned}
& \left|\phi_{w}^{T}(C)\right| \leqq k q^{-(1 / 4 d)\|C\|}, \\
& \left|\phi_{f}^{T}(C)\right| \leqq k q^{-(1 / 8 d)\|C\|},
\end{aligned}
$$

for all clusters. 


\section{Interfaces and Surface Tensions}

In order to define the interfaces and the surface tensions between the different phases we consider the following rectangular boxes:

$\Lambda=\Lambda_{L, M}=\left\{x=\left(x^{1}, \ldots, x^{d}\right) \in \mathbb{Z}^{d} \mid-L / 2 \leqq x^{-1}, \ldots, x^{d-1} \leqq L / 2 ;-M-1 \leqq x^{d} \leqq M\right\}$,

and introduce mixed boundary conditions with respect to an hyperplane $P_{n}$ passing through the origin and orthogonal to a $d$-vector $\mathbf{n}$ (to take into account the angular dependence of the surface tensions). Let $\mathscr{B}_{+}$be the set of bonds having their two endpoints above the plane, i.e. on the set $i^{1} n^{1}+\cdots+i^{d} n^{d} \geqq 0$, and let $\mathscr{B}$ - be the set of bonds having their two endpoints below it, i.e. on the set $i^{1} n^{1}+\cdots+i^{d} n^{d}<0$. We denote by $V$ the set of bonds having their two endpoints inside $\Lambda$ and write

$$
\begin{array}{lll}
\partial_{+} V=\partial V \cap \mathscr{B}_{+}, & \delta_{+} V=\delta V \cap \mathscr{B}_{+}, & \delta_{+} V^{c}=\delta V^{c} \cap \mathscr{B}_{+}, \\
\partial_{-} V=\partial V \cap \mathscr{B}_{-}, & \delta_{-} V=\delta V \cap \mathscr{B}_{-}, & \delta_{-} V^{c}=\delta V^{c} \cap \mathscr{B}_{-} .
\end{array}
$$

The partition function with mixed wired-free boundary condition is:

$$
Z^{(w, f, n)}(V)=\sum_{X \subset V, X \supset \partial_{+} V, X \cap \delta-V^{c}=\varnothing}\left(e^{\beta}-1\right)^{|X|} q^{C_{V}(X)} .
$$

The partition function with mixed wired boundary condition is:

$$
Z^{\left(w, w^{\prime}, n\right)}(V)=\sum_{X \subset V, X \supset \partial_{+}, X \supset \partial_{-} V}^{*}\left(e^{\beta}-1\right)^{|X|} q^{C_{V}(X)}
$$

where the $*$ means that we restrict the sum over sets of bonds such that the connected subset which contains $\partial_{+} V$ is not connected with the connected subset which contains $\partial_{-} V$.

The corresponding surface tensions are defined by

$$
\begin{aligned}
\sigma^{w, f}(\mathbf{n}) & =-(1 / \beta) \lim _{L \rightarrow \infty} \frac{1}{S_{\Lambda}(\mathbf{n})} \lim _{M \rightarrow \infty} \log \frac{Z^{(w, f, \mathbf{n}}(V)}{\left[Z^{w}(V) Z^{f}(V)\right]^{1 / 2}}, \\
\sigma^{w, w^{\prime}}(\mathbf{n}) & =-(1 / \beta) \lim _{L \rightarrow \infty} \frac{1}{S_{\Lambda}(\mathbf{n})} \lim _{M \rightarrow \infty} \log \frac{Z^{\left(w, w^{\prime}, \mathbf{n}\right)}(V)}{Z^{w}(V)},
\end{aligned}
$$

where $S_{\Lambda}(\mathbf{n})$ is the area of the portion inside $\Lambda$ of the hyperplane $P_{n}$. To simplify the notations we introduce

$$
\begin{aligned}
& \sigma^{w, f}=\sigma^{w, f}(\mathbf{n}) \text { for } \mathbf{n}=(0, \ldots, 0,1), \\
& \sigma^{w, f}(\theta)=\sigma^{w, f}(\mathbf{n}) \text { for } \mathbf{n}=(0, \ldots, 0,-\sin \theta, \cos \theta),
\end{aligned}
$$

and the analogous notations for $\sigma^{w, w^{\prime}}$.

3.1. Description of the Order-Disorder Interface. Consider a configuration which contributes to $Z^{(w, f, \mathrm{n})}(V)$. Let $X_{1}$ be the connected component of $X$ which contains $\partial_{+} V$. The co-boundary $\delta X_{1}$ of $X_{1}$ splits into co-connected components. One of them, let it be $\Gamma^{\prime}$, contains the set $\delta_{+} V$, the others (if they exist) are contours of the wired class. We denote $O$ the unique region whose coboundary is $\Gamma^{\prime}$. We define the order-disorder interface $\Gamma$, associated to the configuration $X$, as the co-connected set $\Gamma=\Gamma^{\prime} \backslash \delta_{+} V$, and we define its length as $\|\Gamma\|=|\Gamma|+\Gamma^{(2)} \mid$, where $\Gamma^{(2)}$ are the bonds of $\Gamma$ which contain two endpoints in $O$. The interface $\Gamma$ divides 
the volume $V$ in two regions: the region $O$ above $\Gamma$ corresponding to the ordered phase and the region $D=V \backslash O$ below $\Gamma$ corresponding to the disordered phase.

Proposition 3. The ratio of partition functions entering in the definition of the surface tension $\sigma^{w, f}(\mathbf{n})$, may be written as a sum over all interfaces $\Gamma$ of the following weights;

$$
\begin{aligned}
p_{V}(\Gamma)= & q^{-(1 / 2 d)\|\Gamma\|+1 / 2} \exp \left\{-\sum_{C i \Gamma, C \cap \partial_{+} V=\varnothing} \phi_{w}^{T}(C) \frac{|C \cap O|}{|C|}+\sum_{C i \partial+V} \phi_{w}^{T}(C) \frac{|C \cap D|}{|C|}\right. \\
& -\sum_{C i \Gamma, C \cap \partial_{-} V=\varnothing} \phi_{f}^{T}(C) \frac{|C \cap D|}{|C|}+\sum_{C i \partial_{-} V} \phi_{f}^{T}(C) \frac{|C \cap O|}{|C|} \\
& \left.-\frac{1}{2} \sum_{C i \partial_{+} V, C i \partial_{-} V} \phi_{w}^{T}(C) \frac{|C \cap V|}{|C|}-\frac{1}{2} \sum_{C i \partial+V, C i \partial_{-} V} \phi_{f}^{T}(C) \frac{|C \cap V|}{|C|}\right\},
\end{aligned}
$$

where it is understood that the arguments $C$ of $\phi_{w}^{T}$ and $\phi_{f}^{T}$ are respectively clusters of $w$-contours and clusters of $f$-contours.

Proof. We first notice that for $\phi^{T}$ equal to $\phi_{w}^{T}$ or $\phi_{f}^{T}$ and for any $V \subset \mathscr{B}$, Proposition 2 implies

$$
\begin{aligned}
\sum_{C \subset V} \phi^{T}(C) & =\sum_{b \in V} \sum_{\substack{C \subset V \\
b \in C}} \frac{\phi^{T}(C)}{|C|}=|V| f(\phi)-\sum_{C i \partial V} \frac{\phi^{T}(C)|C \cap V|}{|C|} \\
f(\phi) & =\sum_{b \in C} \frac{\phi^{T}(C)}{|C|} .
\end{aligned}
$$

From the definitions of the sets $O, D$ and $\Gamma$ it follows that

$$
Z^{(w, f, \mathbf{n})}(V)=\sum_{\Gamma} Z^{w}(O) Z^{f}(D),
$$

and therefore (taking again into account Proposition 2)

$$
\begin{aligned}
\log p_{V}(\Gamma)= & \log Z^{w}(O)+\log Z^{f}(D)-(1 / 2) \log Z^{w}(V)-(1 / 2) \log Z^{f}(V) \\
= & |O|\left[\log \left(e^{\beta_{t}}-1\right)+f\left(\phi_{w}\right)\right]+\left(\left|S_{I}(D)\right|+1\right) \log q+|D| f\left(\phi_{f}\right) \\
& -\frac{1}{2}|V|\left[\log \left(e^{\beta_{t}}-1\right)+f\left(\phi_{w}\right)\right]-\frac{1}{2}\left(\left|S_{I}(V)\right|+1\right) \log q-\frac{1}{2}|V| f\left(\phi_{f}\right) \\
& -\sum_{C i \partial O} \phi_{w}^{T}(C) \frac{|C \cap O|}{|C|}-\sum_{C i \partial D} \phi_{f}^{T}(C) \frac{|C \cap D|}{|C|} \\
& +\frac{1}{2} \sum_{C i \partial V} \phi_{w}^{T}(C) \frac{|C \cap V|}{|C|}+\frac{1}{2} \sum_{C i \partial V} \phi_{f}^{T}(C) \frac{|C \cap V|}{|C|} .
\end{aligned}
$$

By applying the identity between free energies stated in Proposition 1, and since $V=O \cup D$, we see that the contribution of the first six terms in the above expression is

$$
\left(\left|S_{I}(D)\right|-\frac{1}{d}|D|-\frac{1}{2}\left(\left|S_{I}(V)\right|-\frac{1}{d}|V|+\frac{1}{2}\right) \log q=\left(-\frac{1}{2 d}\|\Gamma\|+\frac{1}{2}\right) \log q,\right.
$$

where the last equality follows from the corresponding definitions and the fact that $2 d$ bonds meet at every lattice site. It remains to examine the contribution 
of the last four terms and to show that it coincides with the expression inside the exponential stated in the proposition. This is done by rewriting the different contributions according to appropriated and natural decompositions of the sets over which the sums take place and by collecting analogous terms. This ends the proof of the proposition.

3.2. Description of the Order-Order Interface. Consider now a configuration $X$ which contributes to $Z^{\left(w, w^{\prime}, n\right)}(V)$. Then $X$ has at least two different connected components $X_{1}$ and $X_{2}$ such that $X_{1} \supset \partial_{+} V$ and $X_{2} \supset \partial_{-} V$. The co-boundary $\delta X_{1}$ of $X_{1}$ splits into co-connected components. One of them, let it be $\Gamma_{1}^{\prime}$, contains the set $\delta_{+} V$, the others (if they exist) are contours of the wired class. In the same way, the co-boundary $\delta X_{2}$ of $X_{2}$ has one co-connected component $\Gamma_{2}^{\prime}$, which contains the set $\delta_{-} V$. We denote $O=O_{1} \cup O_{2}$ the unique configuration such that the coboundary of $O_{1}$ is $\Gamma_{1}^{\prime}$ and the coboundary of $O_{2}$ is $\Gamma_{2}^{\prime}$. We define the order-order interfaces $\Gamma_{1}, \Gamma_{2}$, associated to the configuration $X$, as the co-connected sets $\Gamma_{1}=\Gamma_{1}^{\prime} \backslash \delta_{+} V$ and $\Gamma_{2}=\Gamma_{2}^{\prime} \backslash \delta_{-} V$. We define their lengths as $\left\|\Gamma_{n}\right\|=\left|\Gamma_{n}\right|+\left|\Gamma_{n}^{(2)}\right|$, where $\Gamma_{n}^{(2)}$ are the bonds of $\Gamma_{n}$ which contain two endpoints in $O_{n}$ and $n=1,2$.

The interfaces $\Gamma_{1}, \Gamma_{2}$ divide the volume $V$ in three regions. The regions $O_{1}$ and $O_{2}$ respectively above $\Gamma_{1}$ and below $\Gamma_{2}$ correspond to the ordered phases, the region $D=V \backslash\left(O_{1} \cup O_{2}\right)$ between them corresponds to the disordered phase.

Proposition 4. The ratio of partition functions entering in the definition of the surface tension $\sigma^{w, w^{\prime}}(\mathbf{n})$, may be written as a sum over all interfaces $\Gamma_{1}, \Gamma_{2}$ of the following weights:

$$
\begin{aligned}
p_{V}\left(\Gamma_{1}, \Gamma_{2}\right)= & q^{-(1 / 2 d)\left(\left\|\Gamma_{1}\right\|+\left\|\Gamma_{2}\right\|\right)+1} \\
& \cdot \exp \left\{-\sum_{C i \Gamma_{1}, C \subset V} \phi_{w}^{T}(C) \frac{\left|C \cap O_{1}\right|}{|C|}-\sum_{C i \Gamma_{2}, C \subset V} \phi_{w}^{T}(C) \frac{\left|C \cap O_{2}\right|}{|C|}\right. \\
& -\sum_{C i \Gamma_{1}} \phi_{f}^{T}(C) \frac{|C \cap D|}{|C|}-\sum_{C i \Gamma_{2}} \phi_{f}^{T}(C) \frac{|C \cap D|}{|C|} \\
& \left.+\sum_{C i V^{c}} \phi_{w}^{T}(C) \frac{|C \cap D|}{|C|}+\sum_{C i \Gamma_{1}, C i \Gamma_{2}} \phi_{f}^{T}(C) \frac{|C \cap D|}{|C|}\right\},
\end{aligned}
$$

where it is understood that the arguments $C$ of $\phi_{w}^{T}$ and $\phi_{f}^{T}$ are respectively clusters of $w$-contours and clusters of $f$-contours.

Proof. From the definitions of the sets $O_{1}, O_{2}, D, \Gamma_{1}$ and $\Gamma_{2}$ we see that

$$
Z^{\left(w, w^{\prime} \cdot \mathrm{n}\right)}(V)=\sum_{\Gamma_{1}, \Gamma_{2}} Z^{w}\left(O_{1}\right) Z^{w}\left(O_{2}\right) Z^{f}(D)
$$

Then, by arguing as in the proof of Proposition 3, we get

$$
\begin{aligned}
\log p_{V}\left(\Gamma_{1}, \Gamma_{2}\right) & =\log Z^{w}\left(O_{1}\right)+\log Z^{w}\left(O_{2}\right)+\log Z^{f}(D)-\log Z^{w}(V) \\
= & \left(\left|O_{1}\right|+\left|O_{2}\right|-|V|\right)\left(\log \left(e^{\beta_{t}}-1\right)+f\left(\phi_{w}\right)\right)+\left(\left|S_{I}(D)\right|+1\right) \log q+|D| f\left(\phi_{f}\right) \\
& -\sum_{C i \partial O_{1}} \phi_{w}^{T}(C) \frac{\left|C \cap O_{1}\right|}{|C|}-\sum_{C i \partial O_{2}} \phi_{w}^{T}(C) \frac{\left|C \cap O_{2}\right|}{|C|} \\
& -\sum_{C i \partial D} \phi_{f}^{T}(C) \frac{|C \cap D|}{|C|}+\sum_{C i \partial V} \phi_{w}^{T}(C) \frac{|C \cap V|}{|C|} .
\end{aligned}
$$


We use the geometric remark

$$
S_{I}(D)-\frac{1}{d}|D|=-\frac{1}{2 d}\left(\left\|\Gamma_{1}\right\|+\left\|\Gamma_{2}\right\|\right) .
$$

Then, the proof of the Proposition follows from similar arguments to those used in the proof of Proposition 3.

\section{Rigidity of the Order-Disorder Interface}

Once one has expressed the weight of the order-disorder interface as in Proposition 3, its rigidity in three or more dimension and for horizontal boundary conditions can be shown in a (rather) standard way, following the method of Dobrushin $[15,16]$ and its generalizations [17-19]. Since there is only a slight difference in the geometry of the interface, compared with the Ising model, we shall only precise how to define the so-called walls and ceilings.

Let $\pi$ denotes the projection parallel to the vertical axis on the horizontal plane: $\pi\left(\left\{x^{1}, \ldots, x^{d}\right\}\right)=\left\{x^{1}, \ldots, x^{d-1}\right\}$, and for any bond $b \in \mathscr{B}$ let $x(b)$ and $y(b)$ denote its endpoints. A bond $b$ of $\Gamma$ is called a ceiling-bond if $\pi(x(b))=\pi(y(b))$ and if there is no other bond $b^{\prime}$ of $\Gamma$ such that $\pi\left(x\left(b^{\prime}\right)\right)=\pi\left(y\left(b^{\prime}\right)\right)=\pi(x(b))$. The bonds of $\Gamma$ which are not ceiling-bonds are called wall-bonds and the set of wall-bonds $W(\Gamma)=\Gamma \backslash \cup(b / b$ is ceiling-bond $)$ is divided in co-connected components called walls.

Then we can follow [15, 17 or 19] to obtain the result. To state it let us introduce some notations. We denote \langle\rangle$^{w, f}$ the expectation of the (infinite volume) Gibbs state with the (horizontal) mixed wired-free boundary conditions and for a fixed bond $b$, we denote $n_{b}$ the occupation number: $n_{b}(X)=1$ if $b$ belongs to $X$ and 0 otherwise. We also denote $x^{d}(b)$ and $y^{d}(b)$ the $d$-coordinates of the endpoints of $b$ and $\mathscr{B}_{+}$denotes the set of bonds $b$ such that $x^{d}(b) \geqq 0$ and $y^{d}(b) \geqq 0$, while $\mathscr{B}_{-}$ denotes the set of bonds $b$ such that $x^{d}(b)<0$ or $y^{d}(b)<0$. Under the above notations we get $([15,17])$.

Theorem 1. Assume that $d \geqq 3$, then there exists a function $g(q)(g(q) \rightarrow 0$ as $q \rightarrow \infty)$ such that

$$
\begin{array}{lll}
\left\langle n_{b}\right\rangle^{w, f}=\operatorname{Prob}\left(n_{b}=1\right) \geqq 1-g(q) & \text { if } & b \in \mathscr{B}_{+}, \\
\left\langle n_{b}\right\rangle^{w, f}=\operatorname{Prob}\left(n_{b}=1\right) \leqq g(q) & \text { if } & b \in \mathscr{B}_{-} .
\end{array}
$$

Moreover one can obtain information about the height of the interface (see $[16,18$ or 19]): for any bond $b$,

$$
\operatorname{Prob}\left(\left|x^{d}(b)\right| \geqq k\right) \leqq \operatorname{cste} e^{-c(q) k},
$$

where $c(q) \rightarrow \infty$ as $q \rightarrow \infty$. On the other hand, the state \langle\rangle$^{w, f}$ is extremal and invariant with respect to horizontal translations.

\section{Restricted Interfaces}

In this section we consider the order-disorder surface tension $\sigma^{w, f}$ which according to Proposition 3 is obtained from the contributions of all order-disorder interfaces 
$\Gamma$ with the associated weights $p_{V}(\Gamma)$. We shall show that only the interfaces having a height less than $L^{2 / 3}$, where $L$ denotes their horizontal size, have a non-negligible contribution to the surface tension.

We first restrict our discussion to the 2-dimensional case. We denote by $\Pi=\Pi(L)$ the vertical strip

$$
\Pi(L)=\left\{(x, y) \in \mathbb{R}^{2}: 0 \leqq x \leqq L\right\},
$$

and by $Q=Q(L) \subset \Pi(L)$ the cigar-shape region

$$
Q(L)=\left\{(x, y) \in \mathbb{R}^{2}: 0 \leqq x \leqq L \text { and }|y-x \operatorname{tg} \theta| \leqq\left[\frac{x(L-x)}{L}\right]^{2 / 3}\right\}, \quad|\theta|<\frac{\pi}{3} .
$$

For simplicity of notation we hereafter denote $V$ the box of Sect. 3 translated along the direction orthogonal to the vector $\mathbf{n}$ in such a way that $V$ is included in $\Pi(L)$, and we denote $p_{\Pi}$ the limit of $p_{V}$ when $M$ goes to infinity.

Main Lemma. Assume that $q$ is large enough and $\beta=\beta_{t}$. Then, for any angle $\theta$ such that $|\theta| \leqq \pi / 3$, we have

$$
\lim _{L \rightarrow \infty} \frac{1}{L} \ln \frac{\sum_{\Gamma \subset Q} p_{\Pi}(\Gamma)}{\sum_{\Gamma \subset \Pi} p_{\Pi}(\Gamma)}=0 .
$$

Before proving the lemma we introduce a slightly more general problem. For each $L$, let $N(L)$ be the integer part of $L \operatorname{tg} \theta$. We denote by $X_{L}$ the point $(L, N(L)) \in \mathbb{Z}^{2}$. We denote by $\Omega_{L}^{\theta}$ the set of all trajectories $\Gamma$ of random lines from 0 to $X_{L}$, which lie entirely inside the strip $\Pi(L)$.

Suppose that for each connected set $C$ of bonds in $\mathbb{Z}^{2}$ and for each $\Gamma \in \Pi(L)$ the function $\varphi(C, \Gamma)$ is given, such that:

2.

$$
\begin{aligned}
& \varphi(C, \Gamma)=0 \text { if } C \cap \Gamma=0 . \\
& \varphi(C, \Gamma) \leqq \exp \{-\lambda d(C)\},
\end{aligned}
$$

where $\lambda \geqq 0$ and $d(C)$ is the length of the shortest connected graph which pass through all boundary bonds of $C$,

3.

$$
\varphi\left(C, \Gamma_{1}\right)=\varphi\left(C, \Gamma_{2}\right) \quad \text { if } \quad \Gamma_{1} \cap \Pi_{C}=\Gamma_{2} \cap \Pi_{C},
$$

where $\Pi_{C}=\left\{(x, y) \in \mathbb{R}^{2}\right.$ :for each $x$ the point $(x, y(x)) \in C$ for some $\left.y(x)\right\}$.

4.

$$
\varphi(C, \Gamma)=\varphi\left(C+t, \Gamma^{1}\right)
$$

for any $t=\left(0, t^{(2)}\right) \in \mathbb{Z}^{2}$, provided $\Gamma^{1} \cap \Pi_{C}=\left(\Gamma \cap \Pi_{C}\right)+t$.

Let us consider the weight

$$
p_{L}(\Gamma)=\lambda^{-|\Gamma|+} \sum_{\cap \cap \Gamma \neq \varnothing} \varphi(C, \Gamma)
$$

and let $\mathscr{P}=\mathscr{P}_{L}$ be the corresponding probability distribution on $\Omega_{L}^{\theta}$. We are going to show that:

Proposition 5. Under the above hypothesis, we have

$$
\mathscr{P}_{L}\{\Gamma \in Q(L)\} \rightarrow 1
$$


when $L \rightarrow \infty$, provided the constant $\lambda$ is large enough.

To show that the Proposition 5 implies the main lemma, we put for any cluster $C$ which intersects $\Gamma$,

$$
\varphi(C, \Gamma)=\left\{\begin{aligned}
\frac{1}{|C|}\left\{-\phi_{w}^{T}(C)|C \cap O|-\phi_{f}^{T}(C)|C \cap D|\right\} \text { if } C \subset \Pi \text { and } C \cap \Gamma \neq \varnothing \\
\frac{1}{|C|}\left\{-\phi_{w}^{T}(C)|C \cap O|+\phi_{f}^{T}(C)|C \cap O|\right\} \\
\text { if } C \cap \partial_{-} \Pi \neq \varnothing, C \cap \partial_{+} \Pi=\varnothing \text { and } C \cap \Gamma \neq \varnothing \\
\frac{1}{|C|}\left\{+\phi_{w}^{T}(C)|C \cap D|-\phi_{f}^{T}(C)|C \cap D|\right\} \\
\text { if } C \cap \partial_{+} \Pi \neq \varnothing, C \cap \partial_{-} \Pi=\varnothing \text { and } C \cap \Gamma \neq \varnothing .
\end{aligned}\right.
$$

Then it is enough to note that the sum of $\left|\phi^{T}(C)\right|$ over those $C$ 's, which contribute to one exponent but not to the other, is uniformly bounded in $\Gamma$ and $L$.

The proof of Proposition 5 is contained in the paper [20] Sect. 4, modulo some minor modifications. For the benefit of the reader we shall give here an outline of it.

The first step is to go from the "canonical ensemble" to the "grand canonical ensemble" $\Omega_{L}^{h}$ which is the set of all trajectories $\Gamma$, starting at $O$, living inside $\Pi_{L}$ and terminating on the line $x=L$. We shall denote this endpoint by $\Gamma(L)$. The statistical weight of a trajectory is given, by definition, by:

$$
P_{L}^{h}(\Gamma)=\lambda^{-|\Gamma|+} \sum_{C \cap \Gamma \neq \varnothing} \varphi(C, \Gamma)+h \Gamma(\Lambda),
$$

where $h$ is some constant, which will depend in some special way on $\theta$. We denote by $\mathscr{P}_{L}^{h}$ the corresponding probability distribution. The connection between the distributions $\mathscr{P}_{L}^{h}$ and $\mathscr{P}_{L}$ is the following: for any $h$ the conditional distribution $\mathscr{P}_{L}^{h}\{\Gamma(L)=N(L)\}$ coincide with $\mathscr{P}_{L}\{\cdot\}$. The choice of $h=h(\theta)$ is made according to the following rule: the difference between $N(L)$ and the expected value of $\Gamma(L)$ according to the distribution $\mathscr{P}_{L}^{h}$, is uniformly bounded in $L$. It is possible to show the existence and uniqueness of such $h(\theta)$.

Let us denote by $Q^{\prime}(L), Q^{\prime \prime}(L)$ the "semi-cigars":

$$
\begin{aligned}
Q^{\prime}(L) & =\left\{(x, y) \in \mathbb{R}^{2}: 0 \leqq x \leqq L \text { and }|y-x \operatorname{tg} \theta| \leqq \frac{1}{2} x^{2 / 3}\right\}, \\
Q^{\prime \prime}(L) & =\left\{(x, y) \in \mathbb{R}^{2}: 0 \leqq x \leqq L \text { and }|y-x \operatorname{tg} \theta| \leqq \frac{1}{2}(L-x)^{2 / 3}\right\} .
\end{aligned}
$$

Then $Q^{\prime}(L) \cap Q^{\prime \prime}(L) \subset Q(L)$. Proposition 5 clearly follows from the two statements:

(i) $\mathscr{P}_{L}^{h}\left\{\Gamma \in Q^{\prime}(L)\right\}=\mathscr{P}_{L}^{h}\left\{\Gamma \in Q^{\prime \prime}(L)\right\} \geqq 1-c L \exp \left\{-c L^{1 / 3}\right\}$

(ii) $\mathscr{P}_{L}^{h}\{\Gamma: \Gamma(L)=N(L)\} \geqq \frac{c}{\sqrt{L}}$

for some constant $c>0$. Indeed,

$$
\mathscr{P}_{L}\{\Gamma \in Q(L)\}=\frac{\mathscr{P}_{L}^{h}\{\Gamma \in Q(L), \Gamma(L)=N(L)\}}{\mathscr{P}_{L}^{h}\{\Gamma(L)=N(L)\}}
$$




$$
\begin{aligned}
& \geqq \frac{\mathscr{P}_{L}^{h}\{\Gamma(L)=N(L)\}+\mathscr{P}_{L}^{h}\{\Gamma \in Q(L)\}-1}{\mathscr{P}_{L}^{h}\{\Gamma(L)=N(L)\}} \\
& \geqq 1-2\left(\frac{c}{\sqrt{L}}\right)^{-1} \cdot c L \exp \left\{-c L^{1 / 3}\right\}
\end{aligned}
$$

which implies Proposition 5.

The statement (ii) is a corollary of the central local limit theorem for the random variable $\Gamma(L)$, which states that

$$
\lim _{L \rightarrow \infty} \sup _{k \in \mathbb{Z}}\left[\operatorname{Var}(\Gamma(L))^{1 / 2} \mathscr{P}_{L}^{h}\{\Gamma(L)=k\}-\frac{1}{\sqrt{2 \pi}} \exp \left\{-\frac{1}{2} \frac{(k-N(L))^{2}}{\operatorname{Var}(\Gamma(L))}\right\}\right]=0
$$

while $\operatorname{Var}(\Gamma(L)) \geqq c_{1} \sqrt{L}$ with $c_{1}>0$.

To study the probability (i) we consider the random variables $\Gamma^{ \pm}(x)$, where $x \in[0, L]$, defined by

$$
\Gamma^{+}(x)=\sup _{(x, t) \in \Gamma}\{t-x \operatorname{tg} \theta\}, \quad \Gamma^{-}(x)=\inf _{(x, t) \in \Gamma}\{t-x \operatorname{tg} \theta\} .
$$

It turns out that for the random variables $\Gamma^{ \pm}(x)$, the central local limit theorem again holds, as $x \rightarrow \infty$. Moreover one can get the estimates on the probabilities for large deviations of $\Gamma^{ \pm}(x)$. The standard idea is the following: it is clear that one has the local limit theorem for $\Gamma^{ \pm}(x)$ not only for $h=h(\theta)$, but for other values of $h$ as well, provided $|h|<1$. Note the following evident relation:

$$
\begin{aligned}
\mathscr{P}_{L}^{h}\left\{\Gamma^{ \pm}(x)=X\right\}= & \mathscr{P}_{L}^{h^{\prime}}\left\{\Gamma^{ \pm}(x)=X\right\} \exp \left\{\left(h-h^{\prime}\right) X\right\} \\
& \cdot\left[\sum_{X \in Z} \exp \left\{\left(h-h^{\prime}\right) X\right\} \mathscr{P}_{L}^{h^{\prime}}\left\{\Gamma^{ \pm}(x)=X\right\}\right]^{-1} .
\end{aligned}
$$

Now we have

$$
\begin{aligned}
\mathscr{P}_{L}^{h}\left\{\Gamma^{ \pm}(x)=X\right\} \leqq & \inf _{h^{\prime}:\left|h^{\prime}\right| \leqq 1} \exp \left\{\left(h-h^{\prime}\right) X\right\} \\
& \cdot\left[\sum_{X \in Z} \exp \left\{\left(h-h^{\prime}\right) X\right\} \mathscr{P}_{L}^{h^{\prime}}\left\{\Gamma^{ \pm}(x)=X\right\}\right]^{-1},
\end{aligned}
$$

which implies the following bounds:

$$
\mathscr{P}_{L}^{h}\left\{\Gamma^{ \pm}(x) \leqq N(X) \pm \frac{L^{2 / 3}}{2}\right\} \leqq \exp \left\{-c L^{1 / 3}\right\}
$$

for any $x \leqq L$ such that $x \geqq X_{0}(h)$, where $X_{0}(h)$ is large enough. Here $c=c(h)$ and the value $X_{0}(h)$ are given by the property that for $x \geqq X_{0}(h)$ the random variable $\Gamma^{+}(x)$ is already well described by normal approximation. The remaining region of finite values of $x$ can be treated by using the simple observation that the curve $\Gamma$ can have stairs in the vicinity of a fixed point, with very small probability.

The proof of the central local limit theorem for $\Gamma(L)$ as well as the estimates on the probabilities of large deviations are based on the fact that the logarithm of the characteristic function $\chi_{\Gamma(L)}^{h}(t)$ of the variable $\Gamma(L)$ is analytic in a certain strip around the real axis. This analyticity in turn is derived from the representation 
of the line $\Gamma$ as a "regular" line with "defects." Representations of that kind were used by Gallavotti [21], Bricmont and Fröhlich [22] and others (see references in [22]). The details can be found in [20].

The analogous result for the 3-dimensional case is limited to the "horizontal" boundary conditions (i.e., $\theta=0$ ). This is because the behaviour of the interface for other boundary conditions is still not known sufficiently well due to the occurrence in that case of infinitely extended defects (see [12]).

The case of horizontal boundary conditions for the 3-dimensional case is in a sense even easier than that of the 2-dimensional case, because here, according with Sect. 4, we know that for large $q$, the interface at the transition point exhibits only finite fluctuations.

\section{Antonov's Rule}

We are now able to prove that Antonov's rule is satisfied at the transition temperature.

Theorem 2. Assume that $q$ is large enough and that $\beta$ is equal to $\beta_{t}$. Then, in two dimensions and for any angle $\theta$ such that $|\theta| \leqq \pi / 3$, we have

$$
\sigma^{w, w^{\prime}}(\theta)=2 \sigma^{w, f}(\theta)>0
$$

and, in three or more dimensions

$$
\sigma^{w, w^{\prime}}=2 \sigma^{w, f}>0
$$

for $\theta=0$.

Proof. As we already mentioned the inequality $\sigma^{w \cdot w^{\prime}}(\mathbf{n}) \geqq 2 \sigma^{w, f}(\mathbf{n})$ was proved in [10]. This result is a consequence of the following correlation inequalities:

$$
\left\langle\prod_{i \in A} \delta\left(\sigma_{i}, a\right) \prod_{i \in \boldsymbol{B}} \delta\left(\sigma_{i}, b\right)\right\rangle \leqq\left\langle\prod_{i \in A} \delta\left(\sigma_{i}, a\right)\right\rangle\left\langle\prod_{i \in \boldsymbol{B}} \delta\left(\sigma_{i}, b\right)\right\rangle,
$$

where free boundary conditions are understood and $A$ and $B$ are finite sets of sites. See [10, 23 and 24] for proofs. The Fortuin-Kasteleyn representation is used in the second reference and the result is valid for real values of $q$.

Let us now prove the converse inequality $\sigma^{w, w^{\prime}}(\theta) \leqq 2 \sigma^{w, f}(\theta)$ at the transition point in the two-dimensional case. We consider the vertical strip $\Pi(L)$ and the cigar-shape region $Q(L)$ introduced in the preceeding section and denote by $Z^{\left(w, w^{\prime}, \theta\right)}(L, M)$ the partition function for the appropriate boundary conditions defined by $\theta$ inside the box $\Lambda \equiv \Lambda_{L, M}$. From Sect. 3 we know that

$$
Z^{\left(w, w^{\prime}, \theta\right)}(L, M) / Z^{w}(L, M)=\sum_{\Gamma_{1} ; \Gamma_{2}} p_{V}\left(\Gamma_{1}, \Gamma_{2}\right) .
$$

Let us restrict the summation to the following set of curves $\Gamma_{1}, \Gamma_{2}$ :

i) The part of the curve $\Gamma_{1}$, lying outside the region $Q_{1}=Q(L-2)+\left(1, L^{3 / 4}\right)$, coincides with the two vertical segments:

$$
\left[\left(\frac{1}{2}, 0\right),\left(\frac{1}{2}, L^{3 / 4}\right)\right] \text { and }\left[\left(L-\frac{1}{2}, L \operatorname{tg} \theta\right),\left(L-\frac{1}{2}, L \operatorname{tg} \theta+L^{3 / 4}\right)\right]
$$


ii) The part of the curve $\Gamma_{2}$, lying outside the region $Q_{2}=Q(L-2)+\left(1,-L^{3 / 4}\right)$, coincides with the two vertical segments:

$$
\left[\left(\frac{1}{2}, 0\right),\left(\frac{1}{2},-L^{3 / 4}\right)\right] \text { and }\left[\left(L-\frac{1}{2}, L \operatorname{tg} \theta\right),\left(L-\frac{1}{2}, L \operatorname{tg} \theta-L^{3 / 4}\right)\right] .
$$

We suppose here that $M \geqq 2 L(1+\operatorname{tg} \theta)$. Let us denote this restricted sum by:

$$
\frac{Z^{\left(w, w^{\prime}, \theta\right)}(L, M \mid Q)}{Z^{w}(L, M)} .
$$

Clearly,

$$
\frac{Z^{\left(w, w^{\prime}, \theta\right)}(L, M)}{Z^{w}(L, M)} \geqq \frac{Z^{\left(w, w^{\prime}, \theta\right)}(L, M \mid Q)}{Z^{w}(L, M)} .
$$

Let $\Gamma_{1}^{\prime}, \Gamma_{2}^{\prime}$ be the parts of the curves $\Gamma_{1}, \Gamma_{2}$ which lie inside the corresponding cigars, and $I_{1}=\Gamma_{1} \backslash \Gamma_{1}^{\prime}, I_{2}=\Gamma_{2} \backslash \Gamma_{2}^{\prime}$. Applying now the Proposition 4, we have

$$
\begin{aligned}
\frac{Z^{\left(w, w^{\prime}, \theta\right)}(L, M \mid Q)}{Z^{w}(L, M)}= & q^{-(1 / 2 d)\left(4 L^{2 / 3}\right)} \sum_{\Gamma_{1}^{\prime} ; \Gamma_{2}^{\prime}} q^{-(1 / 2 d)\left\|\Gamma_{1}^{\prime}\right\|+1 / 2} q^{-(1 / 2 d)\left\|\Gamma_{2}^{\prime}\right\|+1 / 2} \\
& \cdot \exp \left\{-\sum_{C i\left(\Gamma_{1}^{\prime} \cup I_{1}\right), C \subset V} \phi_{w}^{T}(C) \frac{\left|C \cap O_{1}\right|}{|C|}-\sum_{C i\left(\Gamma_{2}^{\prime} \cup I_{2}\right), C \subset V} \phi_{w}^{T}(C) \frac{\left|C \cap O_{2}\right|}{|C|}\right. \\
& -\sum_{C i\left(\Gamma_{1}^{\prime} \cup I_{1}\right)} \phi_{f}^{T}(C) \frac{|C \cap D|}{|C|}-\sum_{C i\left(\Gamma_{2}^{\prime} \cup I_{2}\right)} \phi_{f}^{T}(C) \frac{|C \cap D|}{|C|} \\
& \left.+\sum_{C i V^{c}} \phi_{w}^{T}(C) \frac{|C \cap D|}{|C|}+\sum_{C i\left(\Gamma_{1}^{\prime} \cup I_{1}\right)} \phi_{f}^{T}(C) \frac{|C \cap D|}{|C|}\right\} .
\end{aligned}
$$

Because in the exponent we are interested only in terms of order $L$, we can in the above formula, suppress all segments $I_{1}, I_{2}$, all the clusters $C$ which intersects both $\Gamma_{1}^{\prime}$ and the lower half of $V$, or $\Gamma_{2}^{\prime}$ and the upper half of $V$, and the last term inside the exponential.

After that, the sum $\sum_{\Gamma_{1}^{\prime} ; \Gamma_{2}^{\prime}}$ factorizes, which means that there is no interaction between $\Gamma_{1}^{\prime}$ and $\Gamma_{2}^{\prime}$.

Let us compare now the sum of the remaining terms, corresponding to $\Gamma_{1}^{\prime}$, with the six terms in the exponent in Proposition 3. Their difference is

$$
\begin{aligned}
& -\sum_{C i \Gamma, C i \partial_{-V} V} \phi_{w}^{T}(C) \frac{|C \cap O|}{|C|}+\sum_{C i \Gamma, C i \partial_{-} V} \phi_{f}^{T}(C) \frac{|C \cap D|}{|C|}+\sum_{C i \partial_{-} V} \phi_{f}^{T}(C) \frac{|C \cap O|}{|C|} \\
& +\frac{1}{2} \sum_{C i \partial_{+}, C i \partial_{-} V} \phi_{w}^{T}(C) \frac{|C \cap V|}{|C|}+\frac{1}{2} \sum_{C i \partial_{+} V, C i \partial_{-} V} \phi_{f}^{T}(C) \frac{|C \cap V|}{|C|},
\end{aligned}
$$

modulo some corrections that come because we discarded the contributions of exceptionally long clusters (of the length at least $c L^{3 / 2}$, with some $c>0$ uniformly in $L$ ). But those corrections are uniformly bounded because the functions $\phi_{w}^{T}(C)$, $\phi_{f}^{T}(C)$ decay exponentially in $C$. We claim that each summand in that last sum is 
uniformly bounded. To see it for the first term we have to note that if a cluster $C$ contributes to it, then it must interesect both the boundary $\partial V$ and the boundary $\partial Q$ of the "cigar" $Q(L)$. Because the two lines diverge (as $y^{3 / 2}$, where $y$ is the distance from the origin), and $\phi^{T}(C)$ decays exponentially, the statement follows. The same reasoning applies as well to second and third terms. Also the lines $\partial_{+} V, \partial_{-} V$ diverge, which gives the bounds for the fourth and last terms.

So, up to terms $\exp \{o(L)\}$, we have:

$$
\begin{aligned}
\frac{Z^{\left(w, w^{\prime}, \theta\right)}(L, M \mid Q)}{Z^{w}(L, M)} & =\left[\sum_{\Gamma \subset Q(L)} p_{\Pi}(\Gamma)\right]^{2} \times \exp \{o(L)\} \\
& =\left[\sum_{\Gamma \subset \Pi(L)} p_{\Pi}(\Gamma)\right]^{2} \times \exp \{o(L)\},
\end{aligned}
$$

where in the last step we used the main lemma. From this the result follows.

For the 3-dimensional case, we can repeat the same arguments as in the preceding case. Moreover, we have the freedom of choosing whatever exponents $\alpha, \gamma$ we wish in the definitions of the boxes $Q_{1}$ and $Q_{2}$, with the only restriction that $0<\alpha<\gamma<1$. This is because

$$
\mathscr{P}_{\Pi}\left\{\Gamma:\left|\Gamma^{ \pm}(x)\right|>L^{\alpha} \text { for some } x \in[-L, L] \times[-L, L]\right\} \leqq L^{2} \exp \left(-c L^{\alpha}\right),
$$

and therefore tends to zero when $L \rightarrow \infty$.

Acknowledgements. S. S. would like to thank C. P. T. Marseille for warm hospitality.

\section{References}

1. Baxter, R. J.: Potts model at the critical temperature. J. Phys. C6, L445 (1973); Magnetization discontinuity of the two-dimensional Potts model. J. Phys. A15, 3329 (1982)

2. Kotecký, R., Shlosman, S. B.: First order phase transitions in large entropy lattice systems. Commun. Math. Phys. 83, 493 (1982)

3. Bricmont, J., Kuroda, K., Lebowitz, J. L.: First order phase transitions in lattice and continuous systems. Commun. Math. Phys. 101, 501 (1985)

4. Laanait, L., Messager, A., Ruiz, J.: Phase coexistence and surface tensions for the Potts model. Commun. Math. Phys. 105, 527 (1986)

5. Kotecký, R., Laanait, L., Messager, A., Ruiz, J.: The $q$-state Potts model in the standard Pirogov-Sinai theory: Surface tensions and Wilson loops. J. Stat. Phys. 58, 199 (1990)

6. Laanait, L., Messager, A., Miracle-Sole, S., Ruiz, J., Shlosman, S.: Interfaces is Potts model I: Pirogov-Sinai theory of the Fortuin-Kasteleyn representation. Commun. Math Phys. (in press)

7. Selke, W.: In: Static Critical Phenomena in Inhomogeneous Systems. Pekalski, A., Sznajk (eds.), Lect. Notes in Phys. Vol. 206, Berlin, Heidelberg, New York: Springer 1984

8. Derrida, B., Schick, M.: Interfacial adsorption in multi-state models. J. Phys. A19, 1439 (1986)

9. Bricmont, J., Lebowitz, J.: Wetting in Potts and Blume-Capel models. J. Stat. Phys. 46, 1015 (1987)

10. De Coninck, J., Messager, A., Miracle-Sole, S., Ruiz, J.: A study of perfect wetting in Potts and Blume-Capel models with correlation inequalities. J. Stat. Phys. 52, 45 (1988)

11. Dietrich, S.: Wetting Phenomena. In: Phase Transitions and Critical Phenomena, Vol. 12. Domb, C., Lebowitz, J. L.: (eds), New York: Academic Press 1988

12. Gallavotti, G.: The phase separation line in the two-dimensional Ising model. Commun. Math. Phys 27, 103 (1972) 
13. Bricmont, J., El Mellouki, A., Fröhlich, J.: Random surfaces in Statistical Mechanics: Roughening, rounding and wetting,... J. Stat. Phys. 42, 743 (1986)

14. Kotecký, R., Preiss, D.: Cluster expansion for abstract polymer models. Commun. Math. Phys. 103, 491 (1986)

15. Dobrushin, R. L.: Gibbs states describing the coexistence of phases for a three-dimensional Ising model. Theor. Prob. Appl. 17, 582 (1972)

16. Dobrushin, R. L.: Investigation of Gibbsian states for three-dimensional Lattice systems. Theor. Prob. Appl. 18, 253 (1973)

17. Bricmont, J., Lebowitz, J. L., Olivieri, E., Pfister, D. E.: Non-translation invariant Gibbs states with coexisting phases. I. Commun. Math. Phys. 66, 1 (1979)

18. Bricmont, J., Lebowitz, J. L., Pfister, C. E.: Non-translation invariant Gibbs states with coexisting phases. II. Commun. Math. Phys. 66, 21 (1979)

19. Holický, P. Kotecký, R., Zaharadnick, M.: Rigid interfaces for lattice models at low temperatures. J. Stat. Phys. 50, 755 (1988)

20. Dobrushin, R. L., Kotecky, R., Shlosman, S. B.: Wulff construction: Global shape from local interactions (to appear)

21. Bricmont, J., Fröhlich, J.: Statistical mechanical methods in particle structure analysis of lattice fields theories II: Scalar and surface models. Commun. Math. Phys. 98, 533-578(1985)

22. Schonmann, R. H.: On two correlation inequalities for Potts models. J. Stat. Phys. 52, 61 (1988)

23. Dunlop, F., Laanait, L., Messager, A., Miracle-Sole, S., Ruiz, J.: Multilayer-wetting in partially symmetric $q$-state models. J. Stat. Phys. 59, 1383 (1990)

Communicated by Ya. G. Sinai 